\title{
Commentary Lost in Translation: CRFI Receptor Antagonists and Addiction Treatment
}

\author{
Yavin Shaham, ${ }^{*, 1}$ and Harriet de Wit ${ }^{*, 2}$ \\ 'Behavioral Neuroscience Branch, IRP-NIDA, NIH, Baltimore, MD, USA; ${ }^{2}$ Department of Psychiatry and Behavioral Neuroscience, University of \\ Chicago, IL, USA
}

Neuropsychopharmacology (20 I 6) 4I, 2795-2797. doi: I 0.1038/npp.20 I6.94

In this issue of NPP, Schwandt et al (2016) reported negative results from a comprehensive human translational medicine study in anxious alcohol-dependent women on the effect of the CRFR1 antagonist verucerfont on physiological and psychological stress responses, and subjective craving induced by alcohol-associated cues and stressors. The negative results on craving and stress responses are in agreement with recent work from the same group published in NPP last year (Kwako et al, 2015), using a different CRFR1 antagonist pexacerfont with a less favorable pharmacokinetic profile than verucerfont (Zorrilla et al, 2013). Indeed, the authors chose verucerfont for their second study because studies in rats suggest that the drug should have optimal pharmacokinetic and pharmacodynamic profile in humans due to its slower receptor dissociation rate (Fleck et al, 2012). The improved pharmacokinetic profile of verucerfont was confirmed in the Schwandt et al (2016) study, showing that the drug blocked both neuroendocrine responses to CRF and altered brain activity in response to stimuli with negative emotional valence in alcohol-dependent women. Below we provide a historical perspective for these two studies and discuss their implications for translational addiction research.

In 1981 and 1983, Vale et al. published two papers that have had transformative influence on stress and psychiatry research. In the first study (Vale et al, 1981), they identified a 41-residue ovine hypothalamic peptide that stimulates the hypothalamic-pituitary adrenal axis, leading to the secretion of adrenal corticosterone (or cortisol in humans). In the second study (Swanson et al, 1983), they showed that the new stress-related peptide, termed corticotropinreleasing factor (CRF; the IUPHAR-recommended name; also termed corticotropin-releasing hormone), is expressed in extra-hypothalamic brain areas that control central stress responses (eg, central amygdala, bed nucleus of stria terminalis, prefrontal cortex). The next major advances over the next 20 years were the characterization of the

\footnotetext{
*Correspondence: Dr Y Shaham or H de Wit, IRP-NIDA, Behavioral Neuroscience Branch, 25 I Bayview Blvd, Baltimore, MD 21224, USA, Tel: +443 740 2723, Fax: +443 740 2723,

E-mail: yavin.shaham@nih.gov or hdew@uchicago.edu
}

hypothalamic and extra-hypothalamic CRF systems that include two major receptors (CRFR1 and CRFR2), a CRF binding protein, and the CRF-related peptides urocortin 1, 2, and 3 (Bale and Vale, 2004).

In the psychiatry field, the discovery of the brain CRF systems has led to numerous studies that demonstrated a critical role of these systems in anxiety and depression, as assessed in rodent models (Holsboer and Ising, 2008). These studies have led to intense efforts by several pharmaceutical companies to develop small molecule CRFR antagonists. The first of them, a selective CRFR1 antagonist named CP-154,526 was characterized 20 years ago (Schulz et al, 1996). To date, however, several large clinical studies have failed to demonstrate an efficacy of CRFR1 antagonists in the treatment of anxiety and depression (see references in Schwandt et al (2016), Discussion section).

In the addiction field, CRF was introduced by Koob et al. in a series of seminal studies in the 1980s on the behavioral effects of CRF (Koob and Bloom, 1985) and its role, first in psychostimulant sensitization (Cole et al, 1990), and subsequently in more relevant addiction-related behaviors in animal models: aversive stress-like drug withdrawal symptoms and escalation of drug self-administration (Koob, 2010), as well as stress-induced reinstatement of drug seeking (Mantsch et al, 2016; Shaham et al, 1997). A consistent finding in these studies is that across different drugs of abuse (alcohol, nicotine, cocaine, and heroin), CRFR1 antagonists effectively block aversive symptoms of drug withdrawal, escalation of drug self-administration under extended access conditions, and reinstatement induced by an intermittent footshock stressor, a standard stressor in reinstatement/relapse studies (Koob, 2010; Mantsch et al, 2016). Based on these consistent preclinical findings across drug classes in established animal addiction models, the negative findings from the two human studies with alcoholics are both disappointing and surprising. The main unresolved question is why what appears to be the most promising therapeutic target that has emerged from studies using animal models of addiction during the last 20 years did not show efficacy in humans?

One set of issues to consider is the homology between the laboratory animal model and the human laboratory model 
used to assess the efficacy of the CRFR1 antagonists. In rats, acute stress is known to cause relapse-like return to alcohol seeking (Mantsch et al, 2016); similarly, abstinent alcoholics often report that acutely stressful events cause drug craving and relapse (Sinha et al, 2011). However, the cross-species homology between the two phenomena is not straightforward. Specifically, is exposure to uncontrollable intermittent footshock to a rat comparable to viewing an image with negative emotional content or thinking about one's stressful life events (imaging script) in humans? Are the leverpressing responses used to assess drug seeking in rats comparable to feelings of craving in human drug users? The negative data from the translational medicine studies of Heilig et al. suggest that this may not be the case. However, we believe that the negative human data are more likely due a molecular target failure, specifically related to the human CRF system (which may be fundamentally different from the rodent CRF system), rather than a failure of the animal relapse model. This is because other drugs that are effective in the animal model of stress-induced reinstatement (the alpha-2 adrenoceptor agonists, clonidine and lofexidine) are also effective against stress-induced drug craving in the human laboratory (Mantsch et al, 2016; Sinha et al, 2011).

Another potential rat-human homology consideration is that the negative results of Schwandt et al (2016) were from a study in which the subjects were exclusively females. To our knowledge, studies on the effect of CRFR1 antagonists on escalation of drug taking, aversive stress-like drug withdrawal symptoms, and stress-induced reinstatement were performed in male rats. However, in the previous study (Kwako et al, 2015), Heilig et al. showed that CRFR1 antagonist pexacerfont had no effect on stress-induced alcohol craving and stress responses in a sample composed primarily of male subjects. Thus, it is unlikely that the negative human data are due to the well-known bias in preclinical studies to use male subjects.

A second set of issues to consider is the homology between naturalistic stressful events in the addict's environment and the stress manipulations used in the human laboratory. In the studies of Kwako et al (2015) and Schwandt et al (2016), the methods used to induce stress were standardized, but it is unclear whether they cause the levels of anxiety, craving, or physiological stress responses drug users encounter in their non-laboratory lives. Thus, a significant challenge in human studies is to identify acute stressors that model some of the truly significant stressors faced by drug users in their natural environments like work-related stress, marital crises, and social exclusion (Heilig et al, 2016). Also unknown is the degree to which stress-induced craving and more general craving responses in a non-drug environment (the human laboratory) predict relapse in the addict's drug environment, the ultimate target of novel addiction medications. Some studies suggest that drug craving in the laboratory predict future relapse, (Sinha et al, 2011) whereas other studies do not (Wray et al, 2013).

A third set of issues to consider is the degree to which systemic small molecule CRFR1 antagonists block central CRFR1. Indeed, in the absence of a PET ligand targeting the CRFR1 receptor it is difficult to estimate receptor occupancy after verucerfont administration in humans. However, as discussed above, verucerfont effects on brain activity and neuroendocrine responses to CRF in humans suggest that pharmacokinetic factors cannot account to the negative data of Schwandt et al (2016). Additionally, in the previous study (Kwako et al, 2015) the authors measured CSF levels of pexacerfont, and based on this measure, estimated high central CRFR1 occupancy ( 90\%).

Finally, on the more general question of whether or not CRFR1 antagonists should be considered further for addiction treatment, we would like to point out that the two human studies we discussed were with alcoholic patients. Thus, whether or not these antagonists will decrease stressinduced opiate or psychostimulant craving is a subject for future research. Additionally, the two studies were not designed to test the efficacy of CRFR1 antagonists against two other major addiction-related behaviors for which the antagonists were found effective in the animal models: aversive stress-like drug withdrawal states and escalation of drug intake (Koob, 2010). We suspect that CRFR1 antagonists will not affect stress-related withdrawal symptoms in humans, because in the study of Schwandt et al. (2016) and in another recent publication in NPP (Grillon et al, 2015), verucerfont unexpectedly exacerbated some psychological and physiological stress responses in both alcoholics and normal volunteers. In addition, although early studies demonstrate that it is possible to study escalation of drug use under controlled laboratory conditions (Meyer and Mirin, 1979; Wikler, 1952), it is likely that the effect of CRFR1 antagonist on this important feature of human addiction will not be studied in the future due to ethical considerations related to exposing drug addicts to increasing amounts of abused drugs in the laboratory.

\section{FUNDING AND DISCLOSURE}

The authors declare no conflict of interest. HDW's research is supported by extramural funds from NIDA. YS's research is supported by the Intramural Research Program of NIDA.

\section{REFERENCES}

Bale TL, Vale WW (2004). CRF and CRF receptors: role in stress responsivity and other behaviors. Annu Rev Pharmacol Toxicol 44: 525-557.

Cole BJ, Cador M, Stinus L, Rivier J, Vale W, Koob GF (1990). Central administration of a CRF antagonist blocks the development of stress-induced behavioral sensitization. Brain Res 512: 343-346.

Fleck BA, Hoare SR, Pick RR, Bradbury MJ, Grigoriadis DE (2012). Binding kinetics redefine the antagonist pharmacology of the corticotropin-releasing factor type 1 receptor. I Pharmacol Exp Ther 341: 518-531.

Grillon C, Hale E, Lieberman L, Davis A, Pine DS, Ernst M (2015). The CRH1 antagonist GSK561679 increases human fear but not anxiety as assessed by startle. Neuropsychopharmacology 40: 1064-1071.

Heilig M, Epstein DE, Nader M, Shaham Y (2016). It is time to connect: addiction neuroscience and social context. Nat Rev Neurosci.

Holsboer F, Ising M (2008). Central CRH system in depression and anxiety-evidence from clinical studies with CRH1 receptor antagonists. Eur J Pharmacol 583: 350-357.

Koob GF (2010). The role of CRF and CRF-related peptides in the dark side of addiction. Brain Res 1314: 3-14. 
Koob GF, Bloom FE (1985). Corticotropin-releasing factor and behavior. Fed Proc 44(1 Pt 2): 259-263.

Kwako LE, Spagnolo PA, Schwandt ML, Thorsell A, George DT, Momenan R et al (2015). The corticotropin releasing hormone-1 (CRH1) receptor antagonist pexacerfont in alcohol dependence: a randomized controlled experimental medicine study. Neuropsychopharmacology 40: 1053-1063.

Mantsch JR, Baker DA, Funk D, Le AD, Shaham Y (2016). Stressinduced reinstatement of drug seeking: 20 years of progress. Neuropsychopharmacology 41: 335-356.

Meyer RE, Mirin SM (1979). The Heroin Stimulus: Implications for a Theory of Addiction. Plenum Medical Book Company: New York.

Schulz DW, Mansbach RS, Sprouse J, Braselton JP, Collins J, Corman $\mathrm{M}$ et al (1996). CP-154,526: a potent and selective nonpeptide antagonist of corticotropin releasing factor receptors. Proc Natl Acad Sci U S A 93: 10477-10482.

Schwandt ML, Cortes CR, Kwako LE, George DT, Momenan R, Sinha $\mathrm{R}$ et al (2016). The CRF1 antagonist verucerfont in anxious alcohol dependent women: Translation of neuroendocrine, but not of anti-craving effects. Neuropsychopharmacology.

Shaham Y, Funk D, Erb S, Brown TJ, Walker CD, Stewart J (1997). Corticotropin-releasing factor, but not corticosterone, is involved in stress-induced relapse to heroin-seeking in rats. J Neurosci 17: 2605-2614.

Sinha R, Shaham Y, Heilig M (2011). Translational and reverse translational research on the role of stress in drug craving and relapse. Psychopharmacology (Berl) 218: 69-82.

Swanson LW, Sawchenko PE, Rivier J, Vale WW (1983). Organization of ovine corticotropin-releasing factor immunoreactive cells and fibers in the rat brain: an immunohistochemical study. Neuroendocrinology 36: 165-186.

Vale W, Spiess J, Rivier C, Rivier J (1981). Characterization of a 41-residue ovine hypothalamic peptide that stimulates secretion of corticotropin and beta-endorphin. Science 213: 1394-1397.

Wikler A (1952). A psychodynamic study of a patient during experimental self-regulated re-addiction to morphine. Psychiatr $Q$ 26: 270-293.

Wray JM, Gass JC, Tiffany ST (2013). A systematic review of the relationships between craving and smoking cessation. Nicotine Tob Res 15: 1167-1182.

Zorrilla EP, Heilig M, de Wit H, Shaham Y (2013). Behavioral, biological, and chemical perspectives on targeting $\mathrm{CRF}(1)$ receptor antagonists to treat alcoholism. Drug Alcohol Depend 128: $175-186$. 